\title{
Functional significance of histone deacetylase diversity
}

\author{
Saadi Khochbin", André Verdel, Claudie Lemercier and Daphné Seigneurin-Berny
}

Laboratoire de Biologie Moléculaire et Cellulaire de la Différenciation — INSERM U309, Equipe, Chromatine et Expression des Gènes, Institut Albert Bonniot, Faculté de Médecine, Domaine de la Merci, 38706 La Tronche Cedex, Grenoble, France

${ }^{*}$ Corresponding author

\begin{abstract}
Nucleocytoplasmic shuttling of histone deacetylases is emerging as a major step in determining the composition, and hence the activity, of the corresponding nuclear regulatory complexes. This shuttling process is one of the distinctive characteristics of these enzymes, themselves belonging to structurally and functionally different classes. Considering the specific features of each class of deacetylases, it is possible to determine how each member can contribute to particular cellular functions.
\end{abstract}

Author Keywords: chromatin; transcription; silencing; transport; nucleocytoplasmic shutting

Subject-index terms: Biochemistry; Cancer Biology; Cell biology; Genetics; Evolution; Structural biology

HDAC - histone deacetylase

MADB - matrix-associated deacetylase body

NuRD/NRD - nucleosome remodelling histone deacetylase

\section{Introduction}

Specific lysines present in the tail of core histones, as well as in other cellular and viral proteins, are sites of reversible acetylation. In the case of histones, this post-translational modification appears to constitute a signal [1] that may function in combination with other covalent modifications to generate an epigenetic code. This information is, in turn, interpreted in terms of modified states of chromatin structure and function [2, 3 and 4]. This specific signal is reversed by machinery containing an essential group of enzymes, known as histone deacetylases (HDACs). The understanding of the nature and function of this machinery relies on the identification of the catalytic subunits, as well as on that of the partner proteins. In different species, an increasing number of HDACs are being identified [5], implying that they might be involved in specialized functions. The identification of the first HDAC [6] revealed the existence of a family of proteins in higher eukaryotes related to a known yeast protein, RPD3 [7]. These proteins exhibit a similar domain organization [8] and could be grouped in a class, now known as class I HDACs. As other HDACs, distinct from RPD3, were found in yeast [9], an analogous situation was expected in higher eukaryotes. A search for other HDACs resulted in the discovery of a second class of HDACs related to yeast HDA1, first in mouse [10], and then in human [11 and 12]. Interestingly, another yeast protein, SIR2, was shown recently to be a HDAC and its mammalian homologues have also been identified as HDACs [13, 14 and 15]. Therefore, SIR2-related 
proteins could form a third class of HDACs in higher eukaryotes. The diversity of histone deacetylases has also been observed in plants; indeed, classes other than the well conserved RPD3-related class I HDACs have been characterized, containing enzymes unrelated to RPD3 [16]. The diversity of HDACs strongly suggests that members of each class may be involved in distinct, and perhaps overlapping, functions. The aim of our review is to evaluate how the diversity of HDACs could be linked to distinct functions in the animal kingdom.

\section{Functional diversity in the class I histone deacetylases}

To date, four enzymes, HDAC1, 2, 3 and 8 [5 and 17], are the known members of the class I deacetylases, including different splice variants of HDAC3 [18]. Members of this class contain a wellconserved catalytic domain that in HDAC1, 2 and 3 encompasses almost two thirds of the protein. The remaining carboxy-terminal portion contains the most divergent sequences and is a distinguishing feature among these different members [8]. HDAC1 and HDAC2 were identified as components of two multiprotein complexes known as Sin3/HDAC and NuRD/Mi2/NRD [19]. The catalytic domains of these two HDACs are highly similar [8] and the reason for the simultaneous presence of these two enzymes in different complexes is not clear. The functional differences between HDAC1 and HDAC2 are therefore difficult to assess. Recent studies, however, suggest that they may be involved in distinct cellular functions. In a chicken B cell line, the disruption of HDAC2 resulted in an altered expression of several genes whereas disruption of HDAC1 had little effect on gene expression [20].

HDAC3 appears to be functionally distinct from the first two members. This enzyme was not found in either Sin3/HDAC or NURD/Mi2/NRD core complexes [19] and therefore may not be directly involved in processes controlled by these complexes [21]. Its major function seems to be tightly linked to the activity of nuclear receptor co-repressors. Indeed, increasing evidence points to HDAC3 being a member of the stable core of the SMRT- and N-CoR-containing complexes [22, 23, 24, 25 and 26]. In contrast to HDAC1 and HDAC2, HDAC3 is an essential protein of established chicken B cells as these cells cannot survive without this deacetylase [27]. Moreover, within the class I deacetylases, HDAC3 is the only enzyme known to shuttle between nucleus and cytoplasm and this shuttling process appears to be an essential feature of its function [27].

Functional diversity in the class II histone deacetylases: linkage between their nucleocytoplasmic shuttling and their functions

The class II HDACs, comprising HDACs 4-7, were identified on the basis of their catalytic domain homology to yeast HDA1 protein. They possess several features that distinguish them from the class I members: they are larger (almost twice the size) and the catalytic domain of HDAC4, 5 and 7 is located in the carboxy-terminal half of the protein (Fig. 1). HDAC6 is a unique deacetylase, possessing two catalytic domains [10 and 12] and is probably a functionally distinct member of the family [28]. Some of the functions of HDAC4, 5 and 7 appear to be shared with class I members. Indeed, it has been shown that specific nuclear bodies defined as the MADB (matrix-associated deacetylase body), visualized after over-expression of HDAC4, 5 and 7, contain certain members of NuRD and Sin3 complexes, as well as class I HDACs, HDAC1, 2 and 3. Nuclear receptor co-repressor, SMRT and NCoR have also been found in these bodies [29]. It is therefore possible that HDAC5 and 7 participate, with the class I members, in nuclear-receptor-mediated silencing [30 and 31]. Class II members do not seem to participate, however, in the two major well-characterized deacetylase complexesSin3/HDAC and NuRD/Mi2/NRD. Indeed, none of the class II HDACs was found to interact with the Mi2 proteins [12 and 29] and although there is some evidence for the recruitment of HDAC7 by Sin 3A [30], this HDAC has not been found in the canonical Sin3/HDAC complex [19].

Although a combination of class I and II HDACs are found in nuclear complexes, the involvement of each member in various nuclear functions is dependent on an additional level of regulation controlling their intracellular localization. Indeed, one of the most interesting characteristics of the class II HDACs is the regulated nucleocytoplasmic shuttling of all known members [28, 32, 33 and 34] ( Fig. 2). In specific cell lines, HDAC4 and 5 are retained in the cytoplasm depending on the activity of the 14-3-3 anchoring protein [33 and 34]. Consequently, the nature and the activity of nuclear complexes containing HDAC4 and 5 depend on the intracellular localization of these HDACs. There is at least one example of such a situation: both HDAC4 and 5 interact directly with and repress the transcriptional activity of members of the MEF2 transcription factors [32, 35 and 36], depending on their presence in the nucleus [33 and 34]. HDAC7, which is related to HDAC4 and 5, also appears to control MEF2 transcriptional activity depending on a similar nucleocytoplasmic shuttling process $(\mathrm{H}-\mathrm{Y}$ Kao, personal communication). 
HDAC6 has also been shown to have a regulated intracellular localisation [28]. Although the ectopically-expressed protein, like HDAC4 and HDAC5, is capable of CRM1-dependent nucleocytoplasmic shuttling, however, the endogenous HDAC6 is essentially cytoplasmic and only a fraction of the protein is found in the nucleus after treatment of cells with CRM1-specific inhibitor leptomycin B or upon the arrest of cell proliferation [28]. Moreover, the HDAC activity of HDAC6 was found to be resistant to trapoxin, a well-known inhibitor (M Yoshida, personal communication), strongly suggesting that this enzyme possesses distinct properties.

\section{Class III histone deacetylases: enzymes linking cell metabolism to the control of histone acetylation}

SIR2, a yeast repressor of transcription has been shown recently to have an in vitro $\mathrm{NAD}^{+}$-dependent HDAC activity [13, 14 and 15]. Here again, as with yeast RPD3 and HDA1 [8], a large domain of the protein shows significant sequence homology with a group of prokaryotic enzymes [37 and 38]. Yeast SIR2 can be considered, therefore, as a founding member of a large family of related proteins present in higher eukaryotes that we will refer to here as class III HDACs. A detailed analysis of all the homologous proteins found in higher eukaryotes has revealed the presence of at least four distinct groups. For instance, in humans these groups encompass seven genes, encoding different proteins named SIRT1-7 [38]. Although the discovery of the deacetylase activity of SIR2 is recent and no complexes containing this enzyme have yet been characterized, it can be easily assumed that the class III members are involved in functions not shared with the class I and II deacetylases. First of all, there could be a direct link between their deacetylase activity and the cellular energy and redox states. Second, in contrast to RPD3 and HDA1, a large fraction of the yeast SIR2 protein is found within the nucleolus, associated with the tandem rDNA repeats, where it ensures various critical functions [39]. Third, besides the HDAC activity, another enzymatic activity, ADP-ribosyltransferase, is associated with SIR2 [13 and 40]. All these characteristics suggest that SIR2-related proteins are members of a functionally distinct class of HDACs. The challenge would be now to define the functional specificity of each member. Interestingly, as with class I and class II members, several SIR2-related proteins have been found in the cytoplasm [41 and 42].

\section{Conclusions}

Different members of class I and/or class II HDACs are found together in various nuclear complexes. Unfortunately, up to now there has been no hint regarding the significance of the participation of multiple HDACs in one regulatory complex. One might expect, however, the activity of a specific complex to be dependent on the presence of a particular combination of HDACs. Accordingly, the regulation of the intracellular localization of all the class II HDACs through a controlled nucleocytoplasmic shuttling is highly relevant. 14-3-3 appears to be a key player that regulates the intracellular localization of HDAC4 and HDAC5 [33 and 34] and possibly HDAC7. Interestingly, 14-3-3 proteins may play a similar role in the control of the intracellular localization of Hat1 acetyltransferase [43]. This indirect control of the composition, and hence activity, of nuclear HDAC- and maybe histone acetyltransferase-complexes via 14-3-3 establishes a direct link between two important signalling processes: protein acetylation and phosphorylation. Indeed, in general, 14-3-3 proteins bind ligands containing phosphorylated consensus binding sites and this activity is known to participate in the control of the cell cycle, cell death and mitogenesis [44]. Moreover, the presence of at least one member of the class I, all members of the class II, as well as several members of the class III HDACs in the cytoplasm strongly suggests that, besides their well-documented involvement in chromatin silencing, these enzymes may play distinct and specific roles in this compartment that remain to be elucidated.

\footnotetext{
Update

Endogenous HDAC5 present in the nucleus of $\mathrm{C} 2$ myoblasts is efficiently exported into the cytoplasm upon their differentiation into myotubes. The phosphorylation of HDAC5 by the calcium/calmodulindependent protein kinase, CaMK, is found to play an essential role in the nuclear export of HDAC4 and 5 and therefore in the control of myogenesis [45]. Moreover, this CaMK-dependent phosphorylation of HDAC5 enhances its binding to 14-3-3 and its retention in the cytoplasm [46]. In contrast, the activation of Ras-mitogen-activated protein kinase (MAPK) signal transduction pathway results in an accumulation of HDAC4 in the nucleus of $\mathrm{C} 2 \mathrm{C} 12$ myoblast cells [47]. These findings show that kinases linked to independent signaling pathways govern the intracellular localization of
} 
HDAC4 and 5 and that, depending on the type of kinase involved, the protein can be either cytoplasmic or nuclear.

In contrast to other HDACs, the histone deacetylase activity of HDAC6 is found to be resistant to trapoxin and to a new potent deacetylase inhibitor, CHAP1, whereas, like other HDACs, HDAC6 deacetylase activity is inhibited efficiently by trichostatin $A$ [48].

\section{Acknowledgements}

We are grateful to Hung-Ying Kao for communicating results prior publications and the critical reading of the manuscript. To Joseph Torchia, Xiang-Jiao Yang and Sophie Rousseaux, for the critical reading of this manuscript and helpful suggestions. D Seigneurin-Berny. is a recipient of a post-doctoral fellowship from the 'Association pour la Recherche sur le Cancer', A Verdel is a recipient of a PhD fellowship from the 'Ligue Nationale Contre le Cancer, comité de la Haute Savoie' and C Lemercier is a recipient of a post-doctoral fellowship from Sidaction.

\section{References and recommended reading}

Papers of particular interest, published within the annual period of review, have been highlighted as: $\checkmark$ of special interest

$\checkmark \checkmark$ of outstanding interest

\section{References}

1. B.M. Turner, Decoding nucleosome. Cell 75 (1993), pp. 5-8.

2. B.D. Strahl and C.D. Allis, The language of covalent histone modifications. Nature 403 (2000), pp. $41-45$.

3. B.M. Turner, Histone acetylation and an epigenetic code. Bioessays 22 (2000), pp. 836-845.

4. P. Cheung, C.D. Allis and P. Sassone-Corsi, Signaling to chromatin through histone modifications. Cell 103 (2000), pp. 263-271

5. C.A. Johnson and B.M. Turner, Histone deacetylases: complex transducers of nuclear signals. Semin Cell Dev Biol 10 (1999), pp. 179-188.

6. J. Taunton, C.A. Hassig and S.L. Schreiber, A mammalian histone deacetylase related to the yeast transcriptional regulator Rpd3p. Science 272 (1996), pp. 408-411.

7. D.D. Leipe and D. Landsman, Histone deacetylases, acetion utilization proteins and acetylpolyamine amidohydrolases are members of an ancient protein superfamily. Nucleic Acids Res 25 (1997), pp. 3693-3697.

8. S. Khochbin and A.P. Wolffe, The origin and utility of histone deacetylases. FEBS Lett 419 (1997), pp. $157-160$.

9. S.E. Rundlett, A.A. Carmen, R. Kobayashi, S. Bavykin, B.M. Turner and M. Grunstein, HDA1 and RPD3 are members of distinct yeast histone deacetylase complexes that regulate silencing and transcription. Proc Natl Acad Sci USA93 (1996), pp. 14503-14508.

10. A. Verdel and $S$. Khochbin, Identification of a new family of higher eukaryotic histone deacetylases. J Biol Chem 274 (1999), pp. 2440-2445.

11. W. Fischle, S. Emiliani, M.J. Hendzel, T. Nagase, N. Nomura, W. Voelter and E. Verdin, A new family of human histone deacetylases related to Saccharomyces cerevisiae HDA1p. J Biol Chem 274 (1999), pp. 11713-11720

12. C.M. Grozinger, C.A. Hassig and S.L. Schreiber, Three proteins define a class of human histone deacetylases related to yeast Hda1p. Proc Natl Acad Sci USA 96 (1999), pp. 4868-4873. 
$13 \sqrt{ }$ V. S. Imai, C.M. Armstrong, M. Kaeberlein and L. Guarente, Transcriptional silencing and longevity protein Sir2 is an NAD-dependent histone deacetylase. Nature 403 (2000), pp. 795-800 The deacetylase activity of SIR2 was specific for lysine 16 of histone $\mathrm{H} 4$, lysine 9 and 14 of $\mathrm{H} 3$, thus accounting for different properties of this protein - that is, silencing, recombination suppression and the extension of life..

14. J. Landry, A. Sutton, S.T. Tafrov, R.C. Heller, J. Stebbins, L. Pillus and R. Stenglanz, The silencing protein SIR2 and its homologs are NAD-dependent protein deacetylases. Proc Natl Acad Sci USA 97 (2000), pp. 5807-5811.

15. J.S. Smith, C.B. Brachmann, I. Celic, M.A. Kenna, S. Muhammad, V.J. Starai, J.L. Avalos, J.C. Escalante-semerena, C. Grubmeyer, C. Wolberger and J.D. Boeke, A phylogenetically conserved NAD+ -dependent protein deacetylase activity in the Sir2 protein family. Proc Natl Acad Sci USA 97 (2000), pp. 6658-6663.

16. D. Kölle, G. Brosch, T. Lechner, A. Pipal, W. Helliger, J. Taplick and P. Loidl, Different types of maize histone deacetylases are distinguished by a highly complex substrate and site specificity. Biochemistry 38 (1999), pp. 6769-6773.

17. E. Hu, Z. Chen, T. Fredrickson, Y. Zhu, R. Kirkpatrick, G.F. Zhang, K. Johanson, C.M. Sung and R. Liu, Cloning and characterization of a novel human class I histone deacetylase that functions as a transcription repressor. J Biol Chem 275 (2000), pp. 15254-15264.

18. W.M. Yang, Y.L. Yao, J.M. Sun, J.R. Davie and E. Seto, Isolation and characterization of cDNAs corresponding to an additional member of the human histone deacetylase gene family. $J$ Biol Chem 272 (1997), pp. 28001-28007.

19. P.S. Knoepfler and R.N. Eisenman, Sin meets NuRD and other tails of repression. Cell 99 (1999), pp. $447-450$.

$20 \mathrm{~V}$. Y. Takami, H. Kikuchi and T. Nakayama, Chicken histone deacetylase-2 controls the amount of the $\operatorname{IgM~H}$-chain at the steps of both transcription of its gene and alternative processing of its premRNA in the DT40 cell line. J Biol Chem 274 (1999), pp. 23977-23990 Using gene-targeting techniques, the authors of this paper generated homozygous DT40 mutants cells (a chicken B cell line) devoid of two alleles of the HDAC1 and HDAC2 genes. Two-dimensional PAGE demonstrated a changed pattern of protein expression in cells mutant for HDAC2 but not HDAC1..

21. J. Ahringer, NuRD and SIN3 histone deacetylase complexes in development. Trends Genet 16 (2000), pp. 351-356.

22. Y.D. Wen, V. Perissi, L.M. Staszewski, W.M. Yang, A. Krones, C.K. Glass, M.G. Rosenfeld and E. Seto, The histone deacetylase-3 complex contains nuclear receptor corepressors. Proc Natl Acad Sci USA 97 (2000), pp. 7202-7207.

23. M.G. Guenther, W.S. Lane, W. Fischle, E. Verdin, M.A. Lazar and R. Shiekhattar, A core SMRT corepressor complex containing HDAC3 and TBL1, a WD40-repeat protein linked to deafness. Genes Dev 14 (2000), pp. 1048-1057.

24. J. Li, J. Wang, Z. Nawaz, J.M. Liu, J. Qin and J. Wong, Both corepressor proteins SMRT and NCoR exist in large protein complexes containing HDAC3. EMBO J 19 (2000), pp. 4342-4350.

25. F.D. Urnov, J. Yee, L. Sachs, T.N. Collingwood, A. Bauer, H. Beug, Y.B. Shi and A.P. Wolffe, Targeting of $\mathrm{N}-\mathrm{CoR}$ and histone deacetylase 3 by the oncoprotein v-erbA yields a chromatin infrastructure-dependent transcriptional repression pathway. EMBO J 19 (2000), pp. 4074-4090.

26V. C. Underhill, M.S. Qutob, S.P. Yee and J. Torchia, A novel N-CoR complex contains componenets of the mammalian SWI/SNF complex and corepressor KAP-1. J Biol Chem 275 (2000), pp. 40463-40470 The authors provide evidence for the existence of distinct N-CoR-containing 
complexes encompassing different HDAC content: N-CoR1 complex possessed only HDAC3 and NCoR2 complex contained HDAC1 and HDAC2..

$27 \sqrt{ }$. Y. Takami and T. Nakayama, N-terminal region, C-terminal region, nuclear export signal, and deacetylation activity of histone deacetylase-3 are essential for the viability of the DT40 chicken B cell line. J Biol Chem 275 (2000), pp. 16191-16201 The first evidence for the nucleocytoplasmic shuttling of a class I deacetylase, HDAC3, is provided. Moreover, following their inability to generate a null HDAC3 mutant in DT40 chicken B cells, the authors prepared tetracyclin-responsive HDAC3 cell lines to disrupt the endogenous HDAC3 gene and examine its role. Inhibition of HDAC3 expression in these cells caused apoptotic cell death. Complementation experiments show that the nuclear export signal and the deacetylase activity of the protein were essential for cell viability..

28V. A. Verdel, S. Curtet, M.P. Brocard, S. Rousseaux, C. Lemercier, M. Yoshida and S. Khochbin, Active maintenance of mHDA2/mHDAC6 histone-deacetylase in the cytoplasm. Curr Biol 10 (2000), pp. 747-749 The endogenous HDAC6 is here shown to be essentially cytoplasmic and only a fraction of the protein appeared to shuttle between the nucleus and the cytoplasm. A very potent nuclear export signal present at the amino terminus portion of the protein was found to play an essential role in the control of the cytoplasmic localization of the protein..

29V. M. Downes, P. Ordentlich, H.Y. Kao, J.G. Alvarez and R.M. Evans, Identification of a nuclear domain with deacetylase activity. Proc Natl Acad Sci USA 97 (2000), pp. 10330-10335 A description of the identification of new nuclear cores composed of class I and II histone deacetylases, among other components. Interestingly the integrity of these nuclear bodies depends on histone deacetylase activity of the enzymes contributing to the complex..

30. H.Y. Kao, M. Downes, P. Ordentlich and R.M. Evans, Isolation of a novel histone deacetylase reveals that class I and class II deacetylases promote SMRT-mediated repression. Genes Dev 14 (2000), pp. 55-66.

31. E.Y. Huang, J. Zhang, E.A. Miska, M.G. Guenther, T. Kouzarides and M.A. Lazar, Nuclear receptor corepressors partner with class II histone deacetylases in a Sin3-independent repression pathway. Genes Dev 14 (2000), pp. 45-54.

32. E.A. Miska, C. Karlsson, E. Langley, S.J. Nielsen, J. Pines and T. Kouzarides, HDAC4 deacetylase associates with and represses the MEF2 transcription factor. EMBO J 18 (1999), pp. 5099-5107.

33. C.M. Grozinger and S.L. Schreiber, Regulation of histone deacetylase 4 and 5 and transcriptional activity by 14-3-3-dependent cellular localization. Proc Natl Acad Sci USA 97 (2000), pp. 7835-7840.

34. A.H. Wang, M.J. Kruhlak, J. Wu, N.R. Bertos, M. Vezmar, B.I. Posner, D.P. Bazett-Jones and X.J. Yang, Regulation of histone deacetylase 4 by binding of 14-3-3 proteins. Mol Cell Biol 20 (2000), pp. $6904-6912$.

35. C. Lemercier, A. Verdel, B. Galloo, S. Curtet, M.P. Brocard and S. Khochbin, mHDA1/HDAC5 histone deacetylase interacts with and represses MEF2A transcriptional activity. J Biol Chem 275 (2000), pp. 15594-15599.

36. A.H. Wang, N.R. Bertos, M. Vezmar, N. Pelletier, M. Crosato, H.H. Heng, J. Th'ng, J. Han and X.J. Yang, HDAC4, a human histone deacetylase related to yeast HDA1, is a transcriptional corepressor. Mol Cell Biol 19 (1999), pp. 7816-7827.

37. C.B. Brachmann, J.M. Sherman, S.E. Devine, E.E. Cameron, L. Pillus and J.D. Boeke, The SIR2 gene family, conserved from bacteria to humans, functions in silencing, cell cycle progression, and chromosome stability. Genes Dev 9 (1995), pp. 2888-2902.

38. R.A. Frye, Phylogenetic classification of prokaryotic and eukaryotic Sir2-like proteins. Biochem Biophys Res Commun 273 (2000), pp. 793-798. 
39. L. Guarente, Sir2 links chromatin silencing, metabolism, and aging. Genes Dev 14 (2000), pp. 1021-1026.

40. J.C. Tanny, G.J. Dowd, J. Huang, H. Hilz and D. Moazed, An enzymatic activity in the yeast Sir2 protein that is essential for gene silencing. Cell 99 (1999), pp. 735-745.

41. K. Zemzoumi, D. Sereno, C. Francois, E. Guilvard, J.L. Lemesre and A. Ouaissi, Leishmania major: cell type dependent distribution of a $43 \mathrm{kDa}$ antigen related to silent information regulatory-2 protein family. Biol Cell 90 (1998), pp. 239-245.

42. G. Afshar and J.P. Murnane, Characterization of a human gene with sequence homology to Saccharomyces cerevisiae SIR2. Gene 234 (1999), pp. 161-168.

43. A. Imhof and A.P. Wolffe, Purification and properties of the Xenopus Hat1 acetyltransferase: association with the 14-3-3 proteins in the oocyte nucleus. Biochemistry 38 (1999), pp. 13085-13093.

44. H. Fu, R.R. Subramanian and S.C. Masters, 14-3-3 proteins: structure, function, and regulation. Annu Rev Pharmacol Toxicol 40 (2000), pp. 617-647.

45. T.A. McKinsey, C.L. Zhang, J. Lu and E.N. Olson, Signal-dependent nuclear export of a histone deacetylase regulates muscle differentiation. Nature 408 (2000), pp. 106-111.

46. T.A. McKinsey, C.L. Zhang and E.N. Olson, Activation of the myocyte enhancer factor-2 transcription factor by calcium/calmodulin-dependent protein kinase-stimulated binding of 14-3-3 to histone deacetylase 5. Proc Natl Acad Sci USA 97 (2000), pp. 14400-14405.

47. X. Zhou, V.M. Richon, A.H. Wang, X.J. Yang, R.A. Rifkind and P.A. Marks, Histone deacetylase 4 associates with extracellular signal-regulated kinases 1 and 2 , and its cellular localization is regulated by oncogenic Ras. Proc Natl Acad Sci USA 97 (2000), pp. 14329-14333.

48. R. Furumai, Y. Komatsu, N. Nishino, S. Khochbin, M. Yoshida and S. Horinouchi, Potent histone deacetylase inhibitors built from trichostatin $A$ and cyclic tetrapeptide antibiotics including trapoxin. Proc Natl Acad Sci USA 98 (2001), pp. 87-92. 


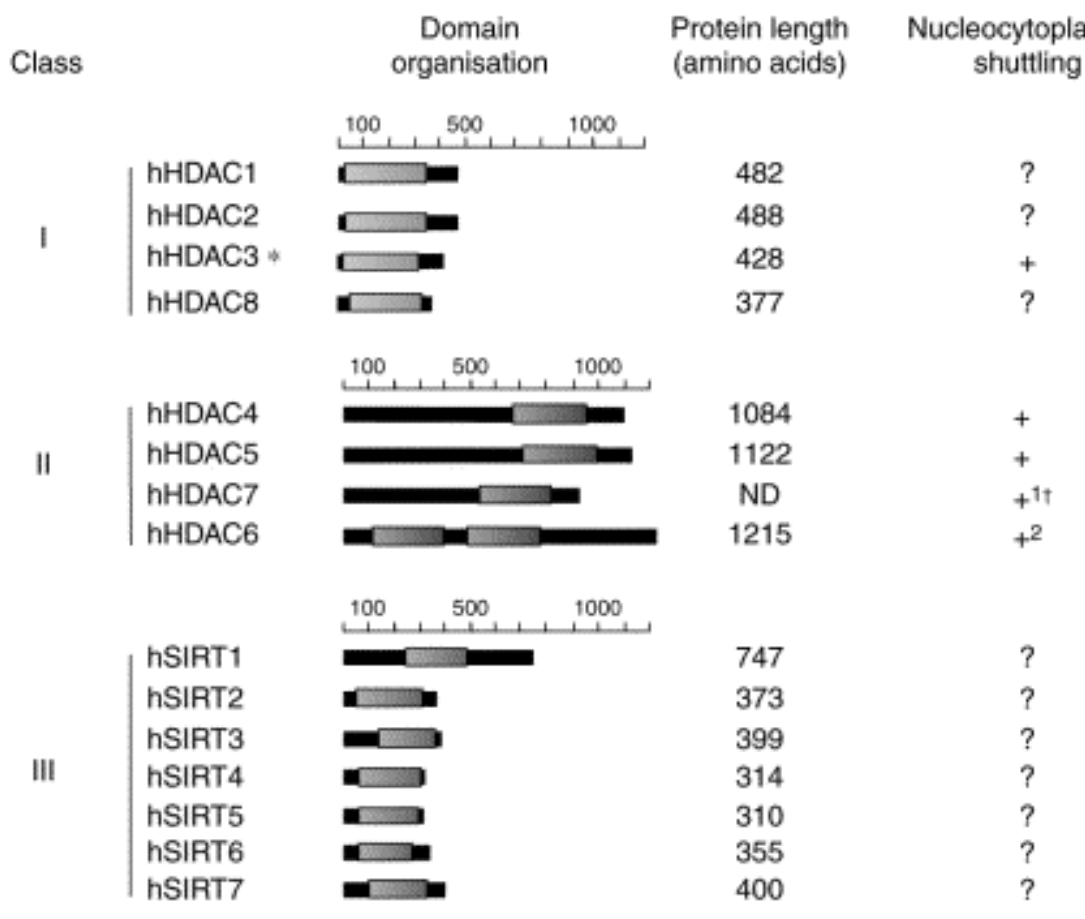

Fig. 1. Identified animal kingdom HDACs can be grouped into three distinct classes each related to a yeast founding member. Class I deacetylases are related to RPD3, class II to HDA1 and class III to SIR2. Boxed regions represent the conserved deacetylase domain. ${ }^{1}$ The shuttling of the ectopically expressed HDAC7 has been observed $(\mathrm{H}-\mathrm{Y}$ Kao, personal communication). ${ }^{2}$ In the case of HDAC6, the endogenous protein has been shown to shuttle between the cytoplasm and the nucleus but this investigation indicated that the protein is essentially cytoplasmic *Variants of HDAC3 have been reported [18]. The sequence of human HDAC7 has not been published. A sequence has, however, been identified as human HDAC7 of 855 aa (GenBank accession number AF239243). ND, not determined. 


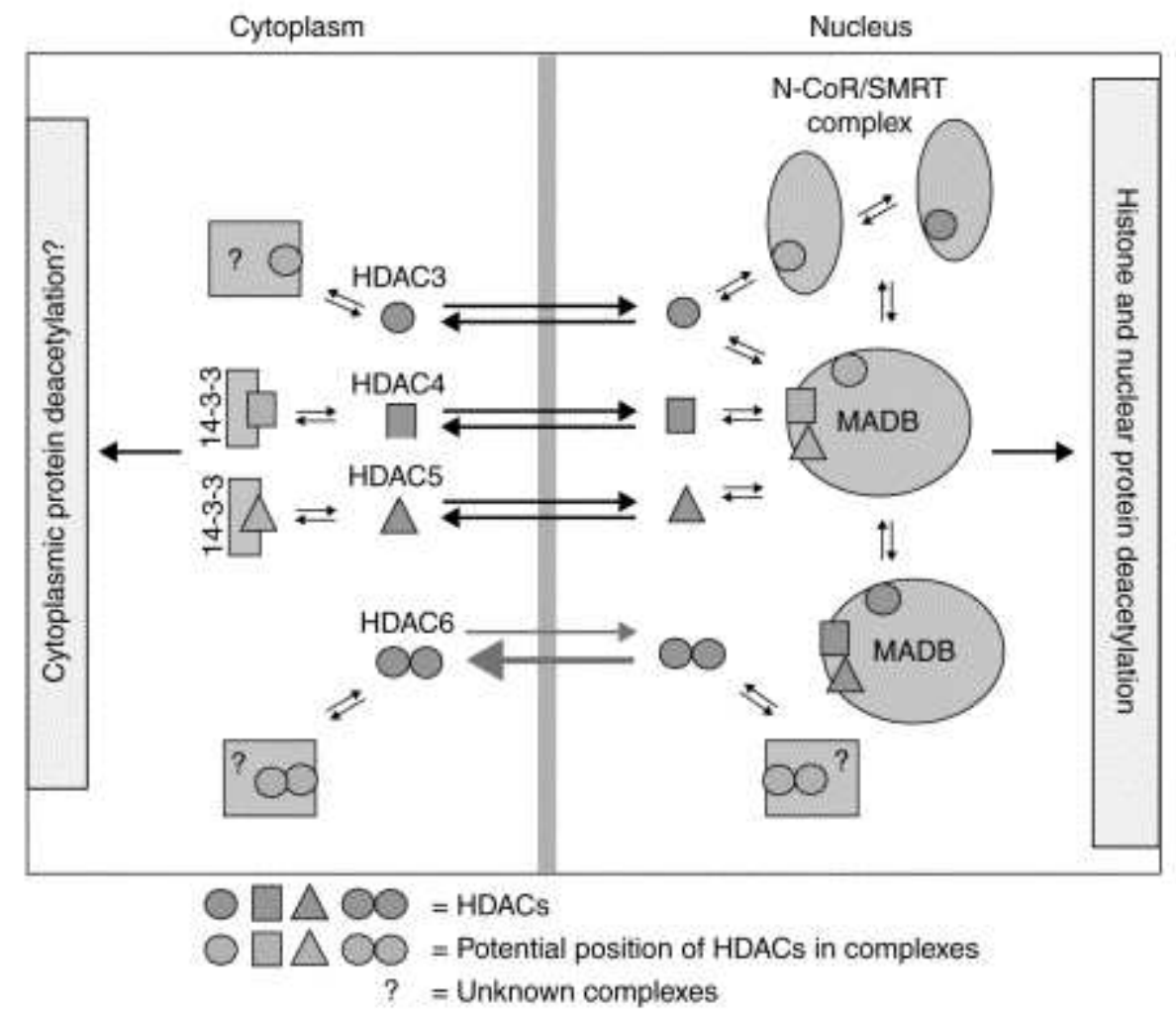

Fig. 2. The specific function of a nuclear deacetylase complex might be dependent on the presence of a particular combination of class I and II HDACs. Accordingly, the nucleocytoplasmic shuttling of histone deacetylases can control their participation in, and hence the activity of, various nuclear complexes. This process specifically links the activity of these nuclear complexes to signals directing 14-3-3/HDAC4 and 5 interactions. The possibility of cytoplasmic protein deacetylation by the shuttling deacetylases should also be considered seriously. In the case of HDAC6, the thick arrow towards the cytoplasm indicates the efficient CRM1-dependent nuclear export of this protein. 